\title{
CLOSEDNESS OF INDEX VALUES FOR SUBFACTORS
}

\author{
DAVID HANDELMAN AND HANS WENZL
}

\begin{abstract}
Let $\left\{A_{i} \subset B_{i}\right\}$ be a collection of inclusions of finite factors with the indices $\left\{\left[B_{i}: A_{i}\right]\right\}$ bounded and each with trivial relative commutant. Then any $W^{*}$ ultraproduct yields an inclusion with trivial relative commutant whose index is the ultralimit of $\left\{\left[B_{i}: A_{i}\right]\right\}$. In particular, the set of values of indices arising from pairs of factors with trivial relative commutant is a closed subset of $\mathbf{R}^{+}$.
\end{abstract}

1. The main result of $[\mathbf{J}]$ asserts that the index of a subfactor of a $\mathrm{II}_{1}$ factor has to be in the set

$$
I=\left\{4 \cos ^{2}(\pi / n) \mid n=3,4, \ldots\right\} \cup\{x \geqslant 4 \mid x \in \mathbf{R}\} .
$$

However, there is still not much known about the set $I_{t}$ of index values for subfactors with trivial relative commutant. All values obtained so far in $I_{t}$ are algebraic integers; moreover, they are the largest eigenvalues of matrices of the form $A^{t} A$ for special nonnegative integer square matrices. (See also the discussion at the end of this section, or [W].)

It had already been shown by Jones that $I_{t}$ is closed under multiplication. We will show among other things that it is topologically closed.

As indicated in the abstract we prove the closedness of $I_{t}$ by showing that ultraproducts preserve the trivial relative commutant property and that the index behaves as it should.

Let $\left\{R_{i}\right\}_{i \in \mathbf{N}}$ be a family of finite $W^{*}$ factors with traces $\left\{\tau_{i}\right\}$, and let $\mathscr{F}$ be a nonprincipal ( $\equiv$ free) ultrafilter on $\mathbf{N}$. Define

$$
\begin{gathered}
R=l^{\infty}\left(R_{i}\right)=\left\{r=\left(r_{i}\right) \mid r_{i} \in R_{i}, \sup \left\|r_{i}\right\|<\infty\right\}, \\
M(\mathscr{F}, R)=\left\{r \in R \mid \forall \text { positive real } \varepsilon,\left\{i \in \mathbf{N} \mid \tau_{i}\left(r_{i} r_{i}^{*}\right)<\varepsilon\right\} \in \mathscr{F}\right\} .
\end{gathered}
$$

Then, as is well known, $R$ is a $W^{*}$ algebra, $M(\mathscr{F}, R)$ is a norm-closed ideal of $R$, and $R_{\infty}$, defined as $R / \mathscr{M}(\mathscr{F}, R)$, is a finite factor. Now let $\left\{A_{i} \subset B_{i}\right\}$ be a family of inclusions of $W^{*}$ factors. Taking the same ultrafilter $\mathscr{F}$ on $\mathbf{N}$, we construct $A_{\infty} \rightarrow B_{\infty}$. However, we may also construct the ultraproduct of $\left\{B_{i}\right\}$ as right $A_{i}$-modules; this is $B / \mathscr{M}(\mathscr{F}, A) \cdot B$.

Received by the editors April 24, 1986 and, in revised form, June 9, 1986.

1980 Mathematics Subject Classification (1985 Revision). Primary 46L10.

The first author was supported by an operating grant and a Steacie Memorical Fellowship from NSERC (Canada).

The second author was supported by Handelman's operating grant.

(1987 American Mathematical Society $0002-9939 / 87 \$ 1.00+\$ .25$ per page 
To compute the index $\left[B_{\infty}: A_{\infty}\right]$, we are going to use the dimension of $B_{\infty}$ as a (finitely generated projective) $A_{\infty}$-module, as in Pimsner and Popa [PP]. For this reason we must show that the ultraproduct of the $\left\{B_{i}\right\}$ as an $A_{\infty}$-module is (naturally) the same as the ultraproduct of the $B_{i}$ as $W^{*}$-factors. In other words, we require

$$
\mathscr{M}(\mathscr{F}, A) B=\mathscr{M}(\mathscr{F}, B)
$$

(notice that one inclusion is obvious). This is a routine deduction from results in [PP].

LEMMA 1. With the notation as above,

$$
\mathscr{M}(\mathscr{F}, A) B=\mathscr{M}(\mathscr{F}, B)
$$

provided $\sup \left[B_{i}: A_{i}\right]<\infty$.

Proof. Since $\mathscr{M}(\mathscr{F}, B)$ is a two-sided ideal of $B$, the inclusion of the left side in the right is clear. Let $n$ be an integer exceeding $\left[B_{i}: A_{i}\right]$ for all $i$. For each $i$, there exists a set of $n$ elements of $B_{i},\left\{m_{j}^{i}\right\}_{j=1}^{n}$, with the properties ascribed to them in [PP, I.3].

Since for $i$ fixed, $\sum_{j}\left(m_{j}^{i}\right)^{*} m_{j}^{i}=\left[B_{i}: A_{i}\right]<n$, we deduce that each of the sequences $M_{j}=\left(m_{j}^{i}\right)_{i \in \mathrm{N}}$ is bounded, so belongs to $l^{\infty}\left(B_{i}\right)=B$. Select $s=\left(s_{i}\right)$ in $\mathscr{M}(\mathscr{F}, B)$. Write $s_{i}=\sum_{j=1}^{n} m_{j}^{i} y_{j 1}$ for some $y_{j i}$ in $A_{i}$. Then $s_{i}^{*} s_{i}=\sum y_{j i}^{*}\left(m_{j}^{i}\right)^{*} m_{k}^{i} y_{k i}$.

Let $E_{i}: B_{i} \rightarrow A_{i}$ be the (onto) expectation, and define the projections $e_{j}^{i}=$ $E_{i}\left(\left(m_{j}^{i}\right)^{*} m_{j}^{i}\right)$ in $A_{i}$. Applying $E_{i}$ to $s_{i}^{*} s_{i}$ we deduce (as $E_{i}\left(\left(m_{j}^{i}\right)^{*} m_{k}\right)=0$ if $\left.j \neq k\right)$, $E\left(s_{i}^{*} s_{i}\right)=\sum_{j} y_{j i}^{*} e_{j}^{i} y_{j i}$. If $e_{j}^{i} \neq 1$, we may replace $y_{j i}$ with $e_{j}^{i} y_{j i}$, by [PP, I.3(4)]. This guarantees that for all $j, \operatorname{tr}\left(y_{j i}^{*} y_{j i}\right) \leqslant \operatorname{tr}\left(s_{i} s_{i}^{*}\right)$ and similarly with the norm. Set $Y_{j}=\left(y_{i j}\right)_{i \in \mathbf{N}}$; as $\left\|Y_{j}\right\| \leqslant \sup \left\|s_{i}\right\|, Y_{j}$ belongs to $l^{\infty}\left(A_{i}\right)=A$. The tracial inequality yields that each $Y_{i}$ belongs to $\mathscr{M}(\mathscr{F}, A)$. Thus

$$
s=\sum M_{j} Y_{j} \in B \mathscr{M}(\mathscr{F}, A) .
$$

As $\mathscr{M}(\mathscr{F}, A), \mathscr{M}(\mathscr{F}, B)$ are stable with respect to ${ }^{*}, s$ belongs to $\mathscr{M}(\mathscr{F}, A) B$ as desired.

Thus the induced map $A_{\infty} \rightarrow B_{\infty}$ is not only one-to-one, but (with notation) from the proof above the $\left\{M_{j}\right\}$ generate $B_{\infty}$ as a right $A_{\infty}$-module, so $B_{\infty}$ is at most $n$-generated as an $A_{\infty}$-module. It follows easily that $B_{\infty}$ is projective as an $A_{\infty}$-module.

By [PP, $\S 1]$, the index is just the dimension of $B_{\infty}$ (as a projective $A_{\infty}$-module). If $p=\left(p_{i}\right)$ is a sequence of projections in $M_{n} A=l^{\infty}\left(M_{n} A_{i}\right)$, then $\tau_{\infty}(p)=$ $\lim _{\mathscr{F}} \tau_{i}\left(p_{i}\right)$, where $\tau_{\infty}$ is the trace on $A$, thus on $M_{n} A$, obtained from $A_{\infty}$ (as in [G2] - this may also be deduced from comparability in $\left.M_{n} A_{\infty}\right)$. As an $A_{i}$-module, $B_{i}$ may be regarded as $P_{i} M_{n} A_{i}$ for some projection, and it follows that

$$
\left[B_{\infty}: A_{\infty}\right]=\lim \left[B_{i}: A_{i}\right] \text {. }
$$

Next, we determine the relative commutant of $A_{\infty}$ in $B_{\infty}$. The following is presumably well known, but we could find no direct reference for it. 
Lemma 2. Let $R$ be a unital $C^{*}$-subalgebra of a finite type II factor $S$, having $\tau$ as its trace. Let \|\|$_{2}$ denote the tracial 2-norm, on $S$. Suppose $s$ is an element of $S$ such that for some real $\delta>0$,

$$
\|s r-r s\|_{2}<\delta \text { for all } r \text { in the unit ball of } R .
$$

Then there exists $z$ in $R^{\prime} \cap S(=\{t \in S \mid t r=r t$ all $r$ in $R\})$ such that $\|s-z\|_{2} \leqslant \delta$.

Proof. Let $C$ be the \|\|$_{2}$-closure of the convex hull of $\left\{u s u^{*} \mid u\right.$ unitary in $\left.R\right\}$. This is also strongly closed (as the set is bounded) and thus weakly closed (as the set is convex), so weakly compact. The group of unitaries of $R$ acts affinely on $C$, so the Ryll-Nardzewski theorem applies (e.g. [Gr, §3.1]). Thus there is a fixed point $z$ in $C ; z$ belongs to $R^{\prime} \cap S$.

If $z_{0}=\sum \lambda_{i} u_{i} s u_{i}^{*}$ with $u_{i}$ unitaries in $R, \lambda_{i}$ positive real numbers with $\sum \lambda_{i}=1$, then

$$
\begin{aligned}
\left\|s-z_{0}\right\|_{2} & =\left\|\sum \lambda_{i}\left(s-u_{i} s u_{i}^{*}\right)\right\|_{2} \leqslant \sum \lambda_{i}\left\|s-u_{i} s u_{i}^{*}\right\|_{2} \\
& =\sum \lambda_{i}\left\|\left(s u_{i}-u_{i} s\right) u_{i}^{*}\right\|_{2}<\sum \lambda_{i} \delta=\delta .
\end{aligned}
$$

As $z$ is the \|\|$_{2}$-closure of such $z_{0}$ 's, $\|s-z\| \leqslant \delta$.

Returning to our initial assumption that $\sup \left[B_{i}: A_{i}\right]<n$, we see that $d_{i}=$ $\operatorname{dim}_{\mathrm{C}} A_{i}^{\prime} \cap B_{i}$ are all less than $n$. Because $d_{i}$ can vary over only finitely many values, there exists a unique integer $d<n$ with $\left\{i \in \mathbf{N} \mid d_{i}=d\right\} \in \mathscr{F}$. We now show that $A_{\infty}^{\prime} \cap B_{\infty}$ has dimension $d$. In particular, if the relative commutants of $A_{i}$ in $B_{i}$ are trivial (i.e., $d_{i}=1$ for all $i$ ) the same is true for that of $A_{\infty}$ in $B_{\infty}$.

Select $b^{0}$ in $A_{\infty}^{\prime} \cap B_{\infty}$. There exists $b=\left(b_{i}\right) \in B$ so that $b+\mathscr{M}(\mathscr{F}, B)=b^{0}$ and $\|b\|=\left\|b^{0}\right\|$. For each $\varepsilon$, the set

$$
I_{\varepsilon}=\left\{i \in \mathbf{N} \mid\left\|r_{i} b_{i}-b_{i} r_{i}\right\|_{2}<\varepsilon \text { for all } r_{i} \text { in the unit ball of } A_{i}\right\}
$$

belongs to $\mathscr{F}$. For each $i$ in $I_{\varepsilon}$, Lemma 2 yields the existence of $z_{i}$ in $A_{i}^{\prime} \cap B_{i}$ with $\left\|b_{i}-z_{i}\right\|_{2} \leqslant \varepsilon$, and we may assume $z_{i}$ is in the unit ball. Thus $Z_{\varepsilon}=\left(z_{i}\right)+\mathscr{M}(\mathscr{F}, B)$ belongs to $A_{\infty}^{\prime} \cap B_{\infty}$ and $\left\|Z_{\varepsilon}-b^{0}\right\|_{2} \leqslant \varepsilon$. Thus $A_{\infty}^{\prime} \cap B_{\infty}$ is the closure of the image of $l^{\infty}\left(A_{i}^{\prime} \cap B_{i}\right)$. As $\left[B_{\infty}: A_{\infty}\right] \leqslant n, A_{\infty}^{\prime} \cap B_{\infty}$ is of finite dimension, so $l^{\infty}\left(A_{i}^{\prime} \cap B_{i}\right)$ maps onto $A_{\infty}^{\prime} \cap B_{\infty}$. It easily follows that $\operatorname{dim} A_{\infty}^{\prime} \cap B_{\infty}=d$ (since if $d_{i} \neq d$, the $i$ th entries may be discarded). A bit more is true: For each dimension $d$, there are only finitely many isomorphism classes of $C^{*}$-algebras of that dimension-hence one class has to occur at the indices constituting a member of $\mathscr{F}$ (while the others do not). So $A_{\infty}^{\prime} \cap B_{\infty}$ is isomorphic to one of the finite dimensional algebras, $A_{i}^{\prime} \cap B_{i}$. To summarize, we have the following.

THEOREM. Let $\left\{A_{i} \subset B_{i}\right\}_{i \in \mathbf{N}}$ be a collection of pairs of finite $W^{*}$-factors with $\sup \left[B_{i}: A_{i}\right]<\infty$. Let $\mathscr{F}$ be a nonprincipal ultrafilter on $\mathbf{N}$, and $A_{\infty} \subset B_{\infty}$ the corresponding inclusions of the $W^{*}$-ultraproducts. Then

(i) $\left[B_{\infty}: A_{\infty}\right]=\lim _{\mathscr{F}}\left[B_{i}: A_{i}\right]$.

(ii) $l^{\infty}\left(A_{i}^{\prime} \cap B_{i}\right)$ maps onto $A_{\infty}^{\prime} \cap B_{\infty}$.

(iii) $\left\{i \in \mathbf{N} \mid A_{i}^{\prime} \cap B_{i} \simeq A_{\infty}^{\prime} \cap B_{\infty}\right\} \in \mathscr{F}$. 
In particular, if $A_{i}^{\prime} \cap B_{i}=\mathbf{C}$ for all $i$, then $A_{\infty}^{\prime} \cap B_{\infty}=\mathrm{C}$. If $\lambda=\lim _{i}\left[B_{i}: A_{i}\right]$ exists, then $\left[B_{\infty}: A_{\infty}\right]=\lambda$.

Corollary. Let $D$ be a finite dimensional $C^{*}$ algebra. Then the subset of $\mathbf{R}^{+}$given by

$\left\{\lambda \mid\right.$ there exists a pair of finite type $W^{*}$-factors $A \subset B$

$$
\text { with } \left.[B: A]=\lambda \text { and } A^{\prime} \cap B \simeq D\right\}
$$

is closed in $\mathbf{R}$.

Of course, the only interesting case is if $A^{\prime} \cap B=\mathrm{C}$, when the set in the Corollary is $I_{t}$. Unfortunately, this does not produce any new values in it. We can however rule out one conjecture about $I_{t}$, namely that it consists precisely of all largest eigenvalues of matrices of the form $A^{t} A$, with $A$ having only nonnegative integer entries. Indeed by an unpublished result of James Shearer (communicated to us by $\mathrm{A}$. Hoffman) the spectral radii of incidence matrices of graphs are dense in $[\lambda, \infty)$, where $\lambda=\tau^{1 / 2}+\tau^{-1 / 2}$ with $\tau$ the golden ratio. As these matrices are symmetric, it follows that the set in this conjecture would be dense in $\left[\lambda^{2}, \infty\right)$. In that case, $I_{t}$ would contain the entire interval, including transcendentals.

It is well known and obvious that $I_{t}$ contains $\mathbf{N}$ and by results of $[\mathbf{J}],\left[I_{t}\right]$ contains $\left\{4 \cos ^{2} \pi / n \mid n=3,4,5, \ldots\right\}$. This was generalized in [W] as follows. Let $k, m$ be positive integers with $1 \leqslant k \leqslant m-2$, and let $\lambda=\left(\lambda_{i}\right)_{i \leqslant k}$ be a young diagram having at most $k$ rows, $\lambda_{i}$ being the length of the $i$ th row. Assume additionally $\lambda_{1}-\lambda_{k} \leqslant m-k$. Then there exists a subfactor of the hyperfinite $\mathrm{II}_{1}$ factor $R$ with trivial relative commutant

$$
\lambda(m)=\prod_{1 \leqslant r<s \leqslant k} \frac{\sin ^{2}\left(\left(\lambda_{r}-\lambda_{s}+s-r\right) \pi / m\right)}{\sin ^{2}((s-r) \pi / m)} .
$$

If $k=2$, and $\lambda=(1)$, the index value will be $4 \cos ^{2} \pi / m$.

It is easy to check that

$$
\lim _{m \rightarrow \infty} \lambda(m)=\prod_{1 \leqslant r<s \leqslant k} \frac{\left(\lambda_{r}-\lambda_{s}+s-r\right)^{2}}{(s-r)^{2}}
$$

which is the square of the dimension of the character of $S U(k)$ with dominant weight $\left(\lambda_{1}-\lambda_{k}, \lambda_{2}-\lambda_{k}, \ldots, \lambda_{k-1}-\lambda_{k}\right)$.

2. Alternate paths. Given the essentially routine nature of all the arguments above, it is not surprising that there are many other methods of proof.

For instance, to show as in [PP, I.3] that if $A \subset B$ are finite factors and ${ }_{A} B$ is finitely generated (i.e., as an $A$-module), then ${ }_{A} B$ is projective follows from [GH, 5.6; and G, 5.18]. To obtain the orthogonal decomposition of [PP, I.3], just apply [BH, III.2.10] to matrices; this yields that any finitely generated closed submodule of a finitely generated projective module over a $C^{*}$ algebra is a direct summand. These direct summands can be orthogonalized as a result of $[\mathbf{K}]$. 
We can give a different proof for the triviality of the relative commutant of $A_{\infty}$ in $B_{\infty}$ by using the following criterion that is also interesting in its own right.

Let $U(S)$ be the unitary group of $S, l(x)$ and $r(x)$ the left resp. right support of $x$ and, if $x$ is normal let $\operatorname{supp}(x)$ be its support.

Lemma 3. Let $R \subset S,[S: R]<\infty$, and $\tau=1 /[S: R]$. Then we have for any projection e in $R$,

$$
\operatorname{tr}\left(\operatorname{supp} E_{R}(e)\right) \leqslant[S: R] \operatorname{tr}(e) .
$$

Proof. If $\operatorname{tr}(e) \geqslant \tau$, the statement is trivial. Note that by [J, 3.1.8] there exists a projection $f$ in $S$ such that $E_{R}(f)=\tau$. Let us just assume $e \leqslant f$. Then

$$
e E_{R}(e) e \leqslant e E_{R}(f) e=\tau e ;
$$

on the other hand, $e E_{R}(e) e \geqslant \tau e$ by [PP, 2.1]. Hence

$$
\tau \operatorname{tr}\left(E_{R}(e)\right)=\tau \operatorname{tr}(e)=\operatorname{tr}\left(e E_{R}(e) e\right)=\operatorname{tr}\left(E_{R}(e)^{2}\right)
$$

As $E_{R}(e) \leqslant \tau$, it follows from spectral calculus that the only nonzero spectral value of $E_{R}(e)$ is $\tau$. Hence $E_{R}(e)=\tau q$ with $q=\operatorname{supp}\left(E_{R}(e)\right)$ and obviously $\operatorname{tr}(q)=$ $1 / \tau \operatorname{tr}(e)$. If $e^{\prime}$ is an arbitrary projection with $\operatorname{tr}\left(e^{\prime}\right) \leqslant \tau$, we can find partial isometries $v$ and $v^{*}$ such that $v v^{*}=e^{\prime}$ and $v^{*} v=e \leqslant f$. In particular $e^{\prime}=v f v^{*}$. By [PP, 1.2] and its proof, there exists $m$ in $R$ such that $m f=v f$ and $e^{\prime}=m f m^{*}$. It follows that $r(m f)=r(v f)=e$ and $e^{\prime}=m e m^{*}$. Hence $E_{R}\left(e^{\prime}\right)=\tau m q m^{*}$ and $\operatorname{supp}\left(E_{R}\left(e^{\prime}\right)\right)=l(m q) \sim r(m q) \leqslant q$.

Proposition. Let $R, S, \tau$ be as in the lemma. Then the following are equivalent:

(i) $R^{\prime} \cap S=\mathrm{C}$.

(ii) For any two projections $p, q$ in $S$ with $p>q$ and $\varepsilon>0$ there exists a unitary $u$ in $R$ such that $\operatorname{tr}\left(u^{*} p u q\right) \geqslant(\tau-\varepsilon) \operatorname{tr}(q)$.

Proof. (ii) $\Rightarrow$ (i) Assume $R^{\prime} \cap S \neq \mathrm{C}$ and let $e$ be a nontrivial projection of $R^{\prime} \cap S$ with $\operatorname{tr}(e) \leqslant 1 / 2$. If we set $q=e$ and $p=1-e$ we obtain $\operatorname{tr}\left(u^{*} p u q\right)=$ $\operatorname{tr}(p q)=0$ for any $u$ in $U(R)$.

(i) $\Rightarrow$ (ii) Let us first assume $\operatorname{tr}(p) \geqslant \tau$. It follows from the Ryll-Nardzewski fixed point theorem that $\delta(p)$, the weak closure of the convex hull of $\left\{u p u^{*} \mid u \in U(R)\right\}$ contains an element $\tilde{p} \in R^{\prime} \cap S=\mathbf{C} 1$. As $\operatorname{tr}$ is constant on $\delta(p), \tilde{p}=\operatorname{tr}(p) \geqslant \tau$. But if $\operatorname{tr}\left(u p u^{*} q\right) \leqslant(\tau-\varepsilon) \operatorname{tr}(q)$ for all $u \in U(R), \operatorname{tr}(p)$ could never be approximated by elements of $\delta(p)$. Note that for any projection $f \in R,\left[S_{f}: R_{f}\right]=[S: R]$ by $\left[\mathbf{J}, 2.1 .4\right.$ and 2.1.7] and $R_{f}^{\prime} \cap S_{f}=\left(R^{\prime} \cap S\right)_{f} \cong$ C. Let $p_{0}=\operatorname{supp} E_{R}(p), q_{0}=$ $\operatorname{supp}\left(E_{R}(q)\right)$. As it is enough to show (ii) for any $u p u^{*}, u$ in $U(R)$, instead of $p$, we can assume either $p_{0} \leqslant q_{0}$ or $q_{0} \leqslant p_{0}$. Note in the first case that

$$
\operatorname{tr}_{s_{u_{0}}}(p)=\frac{\operatorname{tr}(p)}{\operatorname{tr}\left(q_{0}\right)} \geqslant \frac{\operatorname{tr}(q)}{\operatorname{tr}\left(q_{0}\right)} \geqslant \tau
$$

by Lemma 3 . Hence we can find an appropriate unitary in $R_{q_{0}}$ (which can be extended easily to $R$ ). Similarly, we can prove the claim for $q_{0} \leqslant p_{0}$ by reducing by $p_{0}$. 
The constant in (ii) is also the best we can hope for. Let $q$ be a projection in $S$ such that $E_{R}(q)=\tau$ and let $p$ be a projection in $R$ such that $\operatorname{tr}(p)=\tau$. Then

$$
\operatorname{tr}\left(u p u^{*} q\right)=\operatorname{tr}\left(u p u^{*}\left(E_{R}(q)\right)\right)=\tau \operatorname{tr}(p)=\tau \operatorname{tr}(q)
$$

for all $u \in U(R)$.

To show that $A_{\infty} \subset B_{\infty}$ has trivial relative commutant, just observe that we can find for projections $p$ and $q$ in $B_{\infty}$ with $q<p$, sequences $\left(p_{i}\right)$ and $\left(q_{i}\right)$ of projections with $\operatorname{tr}_{i}\left(q_{i}\right) \leqslant \operatorname{tr}_{i}\left(p_{i}\right)$ such that $q=\left(\bar{q}_{i}\right)$ and $p=\left(\bar{p}_{i}\right)$. For any $\varepsilon>0$, we find a unitary $u_{i}$ in $A_{i}$ such that (ii) of the Proposition holds. So $U=\left(u_{i}\right)+$ $\mathscr{M}\left(\mathscr{F}, A_{i}\right)$ is a unitary demonstrating (ii) for $p$ and $q$.

\section{REFERENCES}

[BH] B. Blackadar and D. Handelman, Dimension functions and traces on $C^{*}$-algebras, J. Funct. Anal. 45 (1982), 297-340.

[D] J. Dixmier, Les $C^{*}$-algèbres et leurs représentations, Gauthier-Villars, Paris, 1969.

[G] K. R. Goodearl, Ring theory: Nonsingular rings and modules, Dekker, New York, 1977.

[G2] , Von Neumann regular rings, Pitman, New York, 1979.

[Gr] F. Greenleaf, Invariant means on topological groups, Benjamin, New York.

[GH] K. R. Goodearl and D. E. Handelman, Simple self-injective rings, Comm. Algebra 3 (1975), $797-834$.

[J] V. Jones, Index for subfactors, Invent. Math. 72 (1983), 1-25.

[K] I. Kaplansky, Rings of operators, Benjamin, New York, 1968.

[PP] M. Pimsner and S. Popa, Entropy and index for subfactors, Ann. Sci. École Norm. Sup. (4) 19 (1986), 57-106.

[T] M. Takesaki, Theory of operator algebras. I, Springer-Verlag, Berlin and New York, 1979.

[W] H. Wenzl, Representation of Hecke Algebras and subfactors, Thesis, University of Pennsylvania, 1985.

Department of Mathematics, University of Ottawa, Ottawa, Ontario K1N 6N5, Canada

Department of Mathematics, University of California, Berkeley, California 94720 